\title{
NARMAX Model Identification Using a Set-Theoretic Evolutionary Approach
}

\author{
Jinyao Yana,b,1,*, J.R. Deller, Jr. ${ }^{a, b, 2}$ \\ ${ }^{a}$ Department of Electrical and Computer Engineering, Michigan State University, East Lansing, MI 48824, USA \\ ${ }^{b}$ the NSF BEACON Research Center, Michigan State University, East Lansing, MI 48824, USA
}

\begin{abstract}
A method is presented for identification of system models that are linear in parametric structure, but arbitrarily nonlinear in signal operations. The fundamental parameter estimation task uses a settheoretic analysis of the data to deduce feasible sets of solutions in light of certain model assumptions. In turn, measurable set solution properties are used to assess the viability of nonlinear regressor functions that "compete for survival" as components of the model best fit to represent the system. The strategy blends traditional system identification methods with three modeling strategies that are not commonly employed in signal processing: linear-time-invariant-in-parameters models, set-based parameter identification, and evolutionary selection of the model structure. The algorithm can identify nonlinear model structure and estimate parameters in the presence of different unknown noise scenarios, especially correlated noise. This paper reports the theoretical foundation of the methods and the simulation studies to illustrate the performance benefits.
\end{abstract}

Keywords:

Set-membership estimation, model structure selection, nonlinear system identification, evolutionary algorithm

\section{Introduction}

At its core, signal processing (SP) is not a new discipline. Most of the roughly half-century-long profession is built on a solid foundation of linear mathematics and models that were researched, tested, and refined for several centuries before anyone conceived of an "FFT," or a "digital model" expressed as a "computer program." Yet, SP en-

This work was supported in part by the U.S. National Science Foundation under Cooperative Agreement DBI0939454. Any opinions, conclusions or recommendations expressed are those of the authors and do not necessarily reflect the views of the NSF.

*Corresponding author

Email addresses: yanjinya@egr.msu.edu

(Jinyao Yan), deller@egr.msu.edu (J.R. Deller, Jr.)

${ }^{1}$ Student Member, IEEE and EURASIP

${ }^{2}$ Fellow, IEEE, Member, EURASIP gineers have been brilliantly insightful in shaping, out of this bedrock of mathematics, theories, products, and services that have exploited and synergistically advanced the state of modern networks and digital devices. A rich set of theories and methods based on linear, time-invariant (LTI) models is now familiar to the SP practitioner and it is these LTI models that have largely supported the spectacular technological change we have witnessed over a few short decades. The $21^{\text {st }}$ century SP engineer, however, is increasingly likely to encounter system analysis and design problems in which LTI models are just not sufficient [1]. Biologically-motivated solutions are but one extremely compelling current example of this trend.

Nonlinear and / or time-varying models are difficult around which to design, analyze, and compute solutions. Arduous research over decades 
(again based in classical mathematics) has led to a sufficient body of theoretical and applied knowledge in nonlinear systems to support graduate course offerings at many universities (e.g., [2]). Ad hoc applications of nonlinear systems occupy a small but increasing number of pages in the scholarly literature, an SP-based example being the reemergence of neural network of massive scale in "deep learning" of the structure of speech (e.g., [3]). This progress notwithstanding, the general class of nonlinear models is relatively poorly understood in contrast to the treasury of theoretical and practical LTI knowledge available to the SP practitioner. One approach to accounting for nonLTI system properties, without abandoning the rich SP toolkit for LTI systems is to employ models that are LTI in parameters (LTIiP). For some SP endeavors, nonlinear models that LTIiP are useful and only marginally more difficult to work with than systems with purely LTI properties [4]. It is upon this "bridge" class of nonlinear systems that we shall focus in this paper.

Contemporary linear system theory represents a blend of seminal efforts arising in mathematics and statistics, systems and control engineering, and SP. The model structures employed are often known as "time series models," a name used by early developers of the methods prior to the era of modern SP. The models were initially viewed as structures to explain the spectra of random process realizations (e.g., $[5,6]$ ). With the advent of laboratory-scale computing machines in the 1970s, time series models began to receive significant attention following the publication of the widely-read text by Box and Jenkins [6]. Economists were among the first academicians to have employed these models extensively in their research (e.g., [7]), while the nascent field of SP found applications of the methods in speech processing and spectrum estimation [8, 9, 10, 11]. Even though the early SP work was principally concerned with linear prediction - hence with a "blind input" type of model equivalent to the time-series AR model (see below) - the SP applications represent a subtle shift in the view of the model to one of a system, rather than strictly as a model of process (signal) generation. The name "AR model" was not initially employed by the SP community, but, over the decades, time-series models names have been adopted by many signal and systems engineering researchers to refer to variations of canonical linear constant-coefficient difference equations models with, and sometimes without, stochastic signal components. ${ }^{3}$ Currently, the autoregressive moving average with exogenous input (ARMAX) model, including the special cases of autoregressive (AR), autoregressive moving average (ARMA), and autoregressive with exogenous input (ARX) models, are the most commonly used for linear system identification, which remains a very active research field $[12,13,14]$.

A much wider variety of system nonlinearities leads to a much broader class of potential nonlinear model forms. Different insights, approximations, and application requirements have led to many model structures. Historically, nonlinear system identification has focused on a few specific models that can be tightly defined, albeit limited. Early work, based on the Volterra series, generalizes the linear convolution concept to accommodate nonlinear systems. While different identification methods are still actively studied $[4,15,16]$, system identification based on the Volterra (and related Wiener) series remains a challenging problem in general. The identification of Volterra series often requires restrictive inputs like Gaussian white noise [17] that are not always consistent with applications. Moreover, the Volterra series can require a very large number of parameters to appropriately represent an output process in terms of inputs, even when the physical model is of relatively low order [18]. More recently, research into nonlinear identification has turned to more constrained models including block-structured models, such as the Hammerstein and Wiener models [19, 20, 21]. Due to the simple structure of such models, their identification can be very efficient, but the model forms are limited, and accurate $a$ priori system information is required for satisfactory performance.

\footnotetext{
${ }^{3}$ The cross-disciplinary use of the models has not always sustained a purity of terminology, but we take care in this paper to use the model names in accordance with generally accepted definitions.
} 
The nonlinear-ARMAX (NARMAX) model was introduced by Leontaritis and Billings [22] as a new representation for a wide class of nonlinear systems. As the extension of the linear system ARMAX system, the NARMAX model, of which the nonlinear AR (NAR) and ARX (NARX) models are special cases, ${ }^{4}$ is the most concise and comprehensive representation for nonlinear dynamic system identification [23]. The essence of the NARMAX model is that past outputs are included in the expansions. This makes the model more concise since fewer terms are required to represent systems, but it also means that noise in the output must be taken into account when estimating the model coefficients [18]. The Volterra series, blockstructured models, and many network (e.g., radial basis network, neural network) architectures can be considered special cases of the NARMAX model as well. Identification of NARMAX models, which are applicable to a wide class of nonlinear systems, is of great importance in system modeling.

The NARMAX model can be represented as the LTIiP system form. The most challenging problem in any LTIiP model identification is the determination of the model structure. The procedure must be designed to select the correct model terms (regressor signals) and estimate the model parameters from measurements of system inputs and outputs in the presence of correlated or nonlinearly dependent noise. A simple approach is to estimate a model which includes all of the nonlinear terms and then to prune (backward elimination) the insignificant components[24]. This approach is known to cause numerical and computational problems [18]. Another approach is residual-based selection, in which one term is selected at each time according to some measure of goodness of fit (forward selection) [24]. An example of this approach is the FROLS algorithm proposed by Billings et al. [23] which is based on the orthogonal least squares (OLS) estimator. However, in order for FROLS to achieve unbiased parameter estimation in the presence of colored or more complex noise, the algorithm must repeat-

\footnotetext{
${ }^{4}$ As are, of course, AR, ARX, and ARMAX.
}

edly refit the noise model. Moreover, the forward selection is greedy in only adding one term at a time [25].

In the approach to be presented, the fundamental parameter estimation task uses set-theoretic analysis of the data to deduce feasible sets of solutions in light of certain model assumptions. Several well-known batch and recursive methods can be used to identify point estimates of LTIiP systems, but set-membership algorithms provide sets of feasible parameter vectors rather than a single point estimate $[26,27,28]$. This is achieved through successive refinements of an initial solution set, consistent with a priori constraints on the signal or system model. In the present work, measurable set solution properties are used to assess the viability of nonlinear regressor functions that compete for "survival" as components of the model best suited to represent the system. Specifically, we describe an evolutionary approach to the selection of regressors. The framework presented simultaneously addresses both selection of the model and the parameter estimation. A very significant advantage of the algorithm is the lack of need for assumptions about stationarity or distributional characteristics of the noise. This feature makes the algorithm especially beneficial for NARMAX model identification.

\section{Identification Framework}

Consider a single-input-single-output discretetime system with input $x$ and output $\zeta$, each typically assumed to belong to some well-behaved space, $\mathfrak{X} \subset \mathbb{R}^{\mathbb{Z}}$ (e.g., $\ell_{2}$ ). Let $\mathbb{F}_{\boldsymbol{\theta}}: \mathfrak{X} \rightarrow \mathfrak{X}$ denote the system operator mapping $x$ to $\zeta$, which is parametrized by a real vector $\boldsymbol{\theta} \in \mathfrak{P} \subset \mathbb{R}^{Q}$,

$$
\zeta=\mathbb{F}_{\boldsymbol{\theta}}(x)
$$

The system is said to be linear-in-parameters if, for any $x \in \mathfrak{X}$, for all $\boldsymbol{\theta}, \boldsymbol{\theta}^{\prime} \in \mathfrak{p}$, and for all $\alpha, \alpha^{\prime} \in \mathbb{R}$,

$$
\mathbb{F}_{\alpha \boldsymbol{\theta}+\alpha^{\prime} \boldsymbol{\theta}^{\prime}}(x)=\alpha \mathbb{F}_{\boldsymbol{\theta}}(x)+\alpha^{\prime} \mathbb{F}_{\boldsymbol{\theta}^{\prime}}(x) .
$$

We assume that $\mathbb{F}_{\boldsymbol{\theta}}$ is a continuous operator so that eq. (2) extends to countable additivity (e.g., [29]). 
The internal processing of the system is based on a subset of a candidate set of nonlinear regressor functions, $\Xi_{\varphi}=\left\{\varphi_{q}\right\}$, of size $\left|\Xi_{\varphi}\right|$. Each regressor is a mapping $\varphi_{q}: \mathbb{R}^{r_{q}+s_{q}} \rightarrow \mathbb{R}$, operating on a set of $r_{q}$ past and present system inputs, and $s_{q}$ past outputs. The LTIiP observation model, $\mathbb{O}_{\boldsymbol{\theta}_{*}, \varphi_{*}}$, for $t \in \mathbb{Z}$, is given by

$$
\begin{aligned}
\mathbb{O}_{\boldsymbol{\theta}_{*}, \boldsymbol{\varphi}_{*}}: \quad \zeta[t] & =\sum_{q=1}^{Q} \theta_{q_{*}} \varphi_{q *}\left(x_{-\infty}^{t}, \zeta_{-\infty}^{t-1}\right)+e_{*}[t] \\
& \stackrel{\text { def }}{=} \boldsymbol{\theta}_{*}^{T} \boldsymbol{\varphi}_{*}\left(x_{-\infty}^{t}, \zeta_{-\infty}^{t-1}\right)+e_{*}[t],
\end{aligned}
$$

with $\boldsymbol{\theta}_{*} \in \mathfrak{P}$, and $e_{*} \in \mathbb{R}^{\mathbb{Z}}$ an error sequence (properties described below) representing uncertainties in the model. The " $*$ " subscript indicates a "true," but unknown, quantity associated with the observation model. ${ }^{5}$ The arguments, $x_{-\infty}^{t}$ and $\zeta_{-\infty}^{t-1}$, of the regressor signals $\varphi_{q}$ (or vector $\varphi$ ) indicate that a finite number of elements are selected from the subsequences $\{\ldots, x[t-1], x[t]\}$ and $\{\ldots, \zeta[t-2], \zeta[t-1]\}$ by each $\varphi_{q}$ for processing at time $t$. For conservation of space, we define the vectors of signal samples,

$$
\boldsymbol{u}_{q *}[t] \stackrel{\text { def }}{=}\left[\begin{array}{c}
\text { column vector of } r_{q} \text { inputs from } \\
x_{-\infty}^{t}, \\
\text { and } s_{q} \text { outputs from } \zeta_{-\infty}^{t-1}, \text { used } \\
\text { by } \varphi_{q *} \text { at } t
\end{array}\right]
$$

and the matrix $\boldsymbol{U}_{*}[t]=\left[\begin{array}{lll}\boldsymbol{u}_{1 *}[t] & \cdots & \boldsymbol{u}_{Q *}[t]\end{array}\right]$. Given observations of $x$ and $\zeta$ sufficient to compute outputs on time interval $t=1,2, \ldots, \mathfrak{d}$, we pose an estimation model, formulated as a function of the parameters and regressor signals,

$$
\begin{aligned}
\mathbb{M}_{\boldsymbol{\theta}, \boldsymbol{\varphi}}: \quad \zeta^{p}(t, \boldsymbol{\theta}, \boldsymbol{\varphi}) & =\sum_{q=1}^{Q} \theta_{q} \varphi_{q}\left(\boldsymbol{u}_{q}[t]\right) \\
& \stackrel{\text { def }}{=} \boldsymbol{\theta}^{T} \boldsymbol{\varphi}(\boldsymbol{U}[t])
\end{aligned}
$$

\footnotetext{
${ }^{5}$ Because the index $q$ in $\varphi_{q}$ has been defined as an enumeration of the elements of the candidate set $\Xi_{\varphi}$, the functions in (3) should be indexed as $\varphi_{q_{i} *}, i=1, \ldots, Q$, but we use the slightly abusive notation $\varphi_{q *}$ for simplicity. It is to be understood that $\varphi_{q *}$ is the $q^{\text {th }}$ element selected from $\Xi_{\varphi}$, rather than the $q^{\text {th }}$ element of $\Xi_{\varphi}$.
}

in which each $\varphi_{q}$ is drawn from the set $\Xi_{\varphi}$ (see Footnote 5), $\boldsymbol{\theta} \in \mathfrak{p}$, and the $\boldsymbol{u}_{q}[t]$ and $\boldsymbol{U}[t]$ are defined similarly to (4). The superscript on $\zeta^{p}$ connotes "prediction," as this estimation model corresponds to the classical prediction-error method of Ljung [30] and others. If the observation equation is modeled stochastically, and $\mathcal{E}\left\{e_{*}[t]\right\}=0$ for each $t \in \mathbb{Z}$. The expectation then

$$
\mathcal{E}\left\{\zeta[t] \mid \boldsymbol{\theta}_{*}, \boldsymbol{\varphi}_{*}\right\}=\boldsymbol{\theta}_{*}^{T} \boldsymbol{\varphi}_{*}\left(\boldsymbol{u}_{*}[t]\right)=\zeta^{p}\left(t \mid \boldsymbol{\theta}_{*}, \boldsymbol{\varphi}_{*}\right),
$$

where the "conditioning" notation $\left(\cdots \mid \boldsymbol{\theta}_{*}, \boldsymbol{\varphi}_{*}\right)$ is used in a deterministic function merely to emphasize that the conditioning quantities are to be treated as fixed values. Thus, if the "true" parameters and regressors can be found using the estimation model, the prediction sequence will represent the minimum-mean-square-error (MMSE) estimate of the observed sequence. That is, $\zeta^{p}(t, \boldsymbol{\theta}, \boldsymbol{\varphi})$ is the MMSE predictor of the sequence produced by $\mathbb{O}_{\boldsymbol{\theta}_{*}, \boldsymbol{\varphi}_{*}}$. The prediction residual sequence associated with the general estimation model $\mathbb{M}_{\boldsymbol{\theta}, \boldsymbol{\varphi}}$ is

$$
\begin{aligned}
\varepsilon(t, \boldsymbol{\theta}, \boldsymbol{\varphi})= & \zeta[t]-\zeta^{p}(t, \boldsymbol{\theta}, \boldsymbol{\varphi}) \\
= & \left(\boldsymbol{\theta}_{*}-\boldsymbol{\theta}\right)^{T} \boldsymbol{\varphi}_{*}\left(\boldsymbol{U}_{*}[t]\right)+e_{*}[t] \\
& +\boldsymbol{\theta}^{T}\left[\boldsymbol{\varphi}_{*}\left(\boldsymbol{U}_{*}[t]\right)-\boldsymbol{\varphi}(\boldsymbol{U}[t])\right]
\end{aligned}
$$

indicating error components due to the possible misadjustment in the parameter estimates as well as the possible improper selection of regressor functions. Assuming that the "true" parameters and regressor signals can be found for use in the estimation model, then, eq. (7) reduces to

$$
\varepsilon\left(t \mid \boldsymbol{\theta}=\boldsymbol{\theta}_{*}, \boldsymbol{\varphi}=\boldsymbol{\varphi}_{*}\right)=e_{*}[t],
$$

the asymptotic best-case residual.

The estimation algorithm to be developed has the objective of determining the appropriate regressor signal forms concomitantly with the estimation of parameters in a modeling application. The regressor set is chosen by a genetic algorithm based on an evolutionary view of the selection process. The parameters are identified using a settheoretic approach which supports the evolution by contributing model fitness measures. 


\section{Set-Theoretic Parameter Estimation}

\subsection{Overview of Conventional Methods}

Set-membership (SM) estimation and filtering have been widely researched and broadly applied, but have received significantly more interest and attention among the systems and control research communities. ${ }^{6}$ Recent interest in signal processing is evidenced by publications in this journal $[35,36]$. In this paper we feature the optimal bounding ellipsoid (OBE) algorithms, an important subclass of recursive SM algorithms which have significant application potential in engineering - especially in their extended forms in this work which accommodates wide classes of nonlinear systems. The classic (linear-systems) methods developed here provide enhancements to approaches that are traditionally applied to tasks involving system identification, detection, tracking, and adaptive filtering, and data compression and security. Moreover, the enhanced procedures have the potential to make advances in methods that identify and process nonlinear dynamics. The estimation methods are particularly attractive to SP researchers due to their familiar and readily interpretable structures (e.g., [33]). In this section, we sketch the basic principles of the OBE class of algorithms.

We begin by positing the existence of a "true" observation model of form (3), $\mathbb{O}_{\boldsymbol{\theta}_{*}, \boldsymbol{\varphi}_{*}}$, in which, for the purposes of introducing the OBE algorithm, we assume the "true" regressor signals $\boldsymbol{\varphi}_{q *}\left(\boldsymbol{u}_{q *}[t]\right)$ are known. The "true" parameters, of course, are unknown and to be estimated based on observed values of the sequences $\zeta$ and $x$. Hence, for development purposes only, we use the conditioned estimation model, $\mathbb{M}_{\boldsymbol{\theta} \mid \boldsymbol{\varphi}_{*}}$,

$$
\zeta^{p}\left(t, \boldsymbol{\theta} \mid \boldsymbol{\varphi}_{*}\right)=\boldsymbol{\theta}^{T} \boldsymbol{\varphi}_{*}\left(\boldsymbol{U}_{*}[t]\right)
$$

OBE algorithms are based on the assumption of unknown but bounded disturbances in the observation model. We assume the existence of a sequence of error bounds,

$$
\left|e_{*}[t]\right|<\gamma_{t}, \quad t \in \mathbb{Z}
$$

\footnotetext{
${ }^{6}$ Historical accounts of the field with extensive reference lists are found, for example, in [28, 31, 32, 33, 34].
}

At each $t$, the observation model of eq. (3) in conjunction with the bound $\gamma_{t}$ implies the existence of two hyperplanes in the parameter space $\mathfrak{p} \subset \mathbb{R}^{Q}$,

$$
\begin{aligned}
& \mathcal{H}_{t}^{+}=\left\{\boldsymbol{\theta} \in \mathfrak{P}: \zeta[t]=\boldsymbol{\theta}^{T} \boldsymbol{\varphi}_{*}\left(\boldsymbol{U}_{*}[t]\right)+\gamma_{t}\right\}, \quad \text { and } \\
& \mathcal{H}_{t}^{-}=\left\{\boldsymbol{\theta} \in \mathfrak{P}: \zeta[t]=\boldsymbol{\theta}^{T} \boldsymbol{\varphi}_{*}\left(\boldsymbol{U}_{*}[t]\right)-\gamma_{t}\right\}
\end{aligned}
$$

between which $\boldsymbol{\theta}_{*}$ must lie. The intersection of these pairs of hyperplanes over time forms a convex polytope, say $\overline{\mathbb{P}}_{t} \subset \mathfrak{p}$. The set of points interior to $\overline{\mathbb{P}}_{t}$, say $\mathbb{P}_{t}$, is called the feasibility set, at time $t . \mathbb{P}_{t}$ is the exact set of all parameters in $\mathbb{R}^{Q}$ that could have possibly produced the observed signal using the selected $\mathbb{O}_{\boldsymbol{\theta}_{*}, \boldsymbol{\varphi}_{*}}$ model, while adhering to the assumed constraint on the error bounds, $\gamma_{t}$. The convex polytopes are difficult to track without adding significant complexity to the recursion. However, the existing structure of any OBE-updating procedure readily admits a reasonable set approximation to $\overline{\mathbb{P}}_{t}$ in the form of an hyperellipsoid, say $\overline{\mathbb{E}}_{t}$, which overbounds the polytope $\overline{\mathbb{P}}_{t}$. The set of interior points of $\overline{\mathbb{E}}_{t}$, say $\mathbb{E}_{t}$, called the membership set, is therefore guaranteed to contain the feasibility set $\mathbb{E}_{t} \supseteq \mathbb{P}_{t}$. OBE optimization strategies involve minimization of some measure of the "size" of $\mathbb{E}_{t}$, thus seeking a tight approximation to the feasibility set. Moreover, the updating is very sparse (typically $\ll 10 \%$ of the observations are innovative) leading to a very efficient estimation procedure.

In general, a hyperellipsoidal set in $\mathbb{R}^{Q}$ is a collection of points

$$
\mathbb{E} \stackrel{\text { def }}{=}\left\{\boldsymbol{\theta} \in \mathbb{R}^{Q}:\left(\boldsymbol{\theta}-\boldsymbol{\theta}_{c}\right)^{T} \boldsymbol{K}\left(\boldsymbol{\theta}-\boldsymbol{\theta}_{c}\right)<1\right\}
$$

in which $\boldsymbol{\theta}_{c}$ is the centroid of the set, and $\boldsymbol{K} \in \mathbb{R}^{Q}$ is a nonnegative-definitive matrix. In an $\mathrm{OBE}$ algorithm, the hyperellipsoidal membership set at time $t \geq Q$ is given by

$$
\begin{aligned}
\mathbb{E}_{t} & \stackrel{\text { def }}{=}\left\{\boldsymbol{\theta} \in \mathbb{R}^{Q}:\left(\boldsymbol{\theta}-\boldsymbol{\theta}_{t}\right)^{T} \kappa_{t}^{-1} \boldsymbol{C}_{t}\left(\boldsymbol{\theta}-\boldsymbol{\theta}_{t}\right)<1\right\} \\
& =\left\{\boldsymbol{\theta} \in \mathbb{R}^{Q}:\left(\boldsymbol{\theta}-\boldsymbol{\theta}_{t}\right)^{T} \boldsymbol{C}_{t}\left(\boldsymbol{\theta}-\boldsymbol{\theta}_{t}\right)<\kappa_{t}\right\}
\end{aligned}
$$

in which $\boldsymbol{C}_{t}$ is the sample covariance matrix of 
the observed regressor signals

$$
\boldsymbol{C}_{t} \stackrel{\text { def }}{=} \sum_{n=1}^{t} \boldsymbol{\varphi}(\boldsymbol{U}[n]) \boldsymbol{\varphi}^{T}(\boldsymbol{U}[n])
$$

$\kappa_{t}$ is a positive scalar, and $\boldsymbol{\theta}_{t}$ is the ellipsoid center which can be used as a point estimator of $\boldsymbol{\theta}_{*}$ if desired. Because hyperellipsoidal membership set is structured using second-order statistics, the updating recursions for the OBE-class algorithms are closely related to the weighted recursive least squares (WRLS) algorithm [31]. The recursive updating equations for a generic OBE algorithm [31] are as follows. With $\boldsymbol{P}_{t} \stackrel{\text { def }}{=} \boldsymbol{C}_{t}^{-1}$ (functional dependencies other than that on $t$ are suppressed), for $\mathrm{t}=1,2, \ldots$,

$$
\begin{aligned}
G_{t} & =\boldsymbol{\varphi}_{*}[t]^{T} \mathbf{P}_{t-1} \boldsymbol{\varphi}_{*}[t] \\
\varepsilon[t] & =\zeta[t]-\boldsymbol{\theta}_{t-1}^{T} \boldsymbol{\varphi}_{*}[t] \\
\mathbf{P}_{t} & =\frac{1}{\alpha_{t}}\left[\mathbf{P}_{t-1}-\frac{\lambda_{t} \mathbf{P}_{t-1} \boldsymbol{\varphi}_{*}[t] \boldsymbol{\varphi}_{*}[t]^{T} \mathbf{P}_{t-1}}{\alpha_{t}+\lambda_{t} G_{t}}\right] \\
\boldsymbol{\theta}_{t} & =\boldsymbol{\theta}_{t-1}+\lambda_{t} \mathbf{P}_{t} \boldsymbol{\varphi}_{*}[t] \varepsilon[t], \\
\kappa_{t} & =\alpha_{t} \kappa_{t}+\lambda_{t} \gamma_{t}^{2}-\frac{\alpha_{t} \lambda_{t} \varepsilon^{2}[t]}{\alpha_{t}+\lambda_{t} G_{t}},
\end{aligned}
$$

$\left\{\alpha_{t}\right\}$ and $\left\{\lambda_{t}\right\}$ are nonnegative weighting sequences. The process is typically initialized with $\boldsymbol{\theta}_{0}=0, \kappa_{0}=$ 1 and $\mathbf{P}_{0}=\frac{1}{\mu} \mathbf{I}$ ( $\mu$ is a small number).

Different members of the OBE family are distinguished by the forms of the weighting sequences $\left\{\alpha_{t}\right\}$ and $\left\{\lambda_{t}\right\}$, and the criteria by which optimal values of these weights are determined. Two of the three optimization criteria used in the development of OBE algorithms are directly related to the "size" of $\mathbb{E}_{t}$ at each t. These are: (1) to minimize the determinant of the inverse ellipsoid matrix, $\mu_{t}^{\text {vol }} \stackrel{\text { def }}{=} \operatorname{det}\left\{\kappa_{t} \mathbf{P}_{\mathbf{t}}\right\}$, which is proportional to the square of the volume of the ellipsoid; (2) to minimize the trace of the inverse ellipsoid matrix, $\mu_{t}^{\text {tr }} \stackrel{\text { def }}{=} \operatorname{tr}\left\{\kappa_{t} \mathbf{P}_{\mathbf{t}}\right\}$ which is proportional to the sum of squares of the ellipsoid's semi-axes; and, (3) to minimize the parameter $\kappa_{t}$ which is guaranteed to reduce ellipsoid volume though not to the minimum possible, and which has complex interpretations described in, for example, [35, 36, 37].

Optimization in each iteration generally consists of solving a quadratic function to determine the existence of optimal weights $\lambda_{t}$ in the sense of shrinking the ellipsoid size. If such weights do not exist, the observation can be discarded and the effort of updating avoided. Efficient checks for innovation in the data can be used to circumvent the computation involved in direct solution for weights (e.g., [28, 33, 31]). The process is mildly dependent on the particular OBE strategy used, and the details are carefully documented in the cited references.

In addition to the very sparse and efficient updating procedure used by OBE methods, a significant advantage for application is the lack of need for assumptions about stationarity or distributional characteristics of the error sequence $e_{*}$. Further, OBE algorithms yield a feasible set of solutions that can be exploited (see below), whereas the centroid of the set provides a conventional estimate which is interpretable as a weighted least square error estimate.

Obtaining the advantages of the OBE algorithm requires knowledge of noise bounds which are unavailable in many applications. This issue is particularly poignant in the present deployment of the method because shifting sets of regressor signals in $\mathbb{O}_{\boldsymbol{\theta}_{*}, \boldsymbol{\varphi}_{*}}$ can have significant effects on the error bounds in the model. In the analysis above, we have assumed known regressor signals. We next introduce innovations into the OBE structure to account for the potential for erroneous error bounds as the model undergoes "evolution."

\subsection{Technical Adjustments for Evolving Regres- sor Signals}

To mitigate the problem of unknown error bounds, we incorporate the automatic bound estimation (ABE) procedure [38]. ABE has been studied in a probabilistic setting in which conditions have been proven for stochastic convergence of OBE methods that use the method. The basis for $A B E$ is that, starting with overestimated error bounds, there exists, with nonzero probability, an interval $\mathbb{I}$ of any length $\mathcal{N}$ over which no update takes place. Empirically, the need to adjust the bound is indicated by a sufficiently long period 
without updates ${ }^{7}$. When such an interval occurs, $\mathrm{ABE}$ invokes a bound re-estimation recursion,

$$
\gamma_{t}= \begin{cases}\gamma_{t-1}-d_{J}, & \text { if } d_{J}>0 \\ \gamma_{t-1}, & \text { otherwise }\end{cases}
$$

where, $\quad d_{J}=\left(\kappa_{J-1}\right) G_{J} / Q-\epsilon\left(2 \sqrt{\gamma_{t-1}}-\epsilon\right)$

$$
\text { and } \quad J=\underset{t \in \mathbb{I}}{\arg \max } \varepsilon^{2}[t]
$$

in which $\epsilon$ is a small number, and $\gamma_{t}$ denotes the sequence of estimated bounds with $\gamma_{0}>\gamma_{*}$ (true bound).

Furthermore, an immediate and simple check for the ellipsoid regularity is incorporated into the algorithm. For meaningful parameter estimation, the ellipsoid $\mathbb{E}$ must have non-negative volume. In turn, this condition requires that $\kappa_{t}>0$. The generalized method, $O B E$ with evolved regressor signals (OBE-ERS), is given in Algorithm 1 . While the enhancements can be incorporated into any OBE algorithm, here we employ the OBE algorithm version known as setmembership-stochastic approximation (SM-SA). In the SM-SA algorithm, the volume of the ellipsoid (proportional to $\operatorname{det}\left\{\kappa_{t} P_{t}\right\}$ ) is minimized at each iteration by letting $\lambda_{t}=\beta_{t}$ and $\alpha_{t}=1-\beta_{t}$ in eq. (15), and seeking the optimal $\beta_{t}$ in light of the current measurements [31].

Several evolutionary fitness measures can be derived from the final membership set. For instance, the conventional prediction error energy associated with the centroid of the set (point estimator) can be calculated as a quality measure. Additionally, the estimated bounds and the setrelated-properties, such as the volume of the ellipsoid, and the shape of the ellipsoid, can be used as measures of viability. Naturally, the best fitting model should have the minimum estimation error, the smallest ellipsoid volume, and the smallest estimated error bound. A good model will also arise from an ellipsoidal set of "balanced dimensions," so that no redundant regressors are included in

${ }^{7}$ The OBE-ABE has been proven for stochastic convergence [38]. In application, large data samples $M$ and $\mathcal{N}$ $(=\sqrt{M}$, for example) are chosen to achieve high probability convergence.

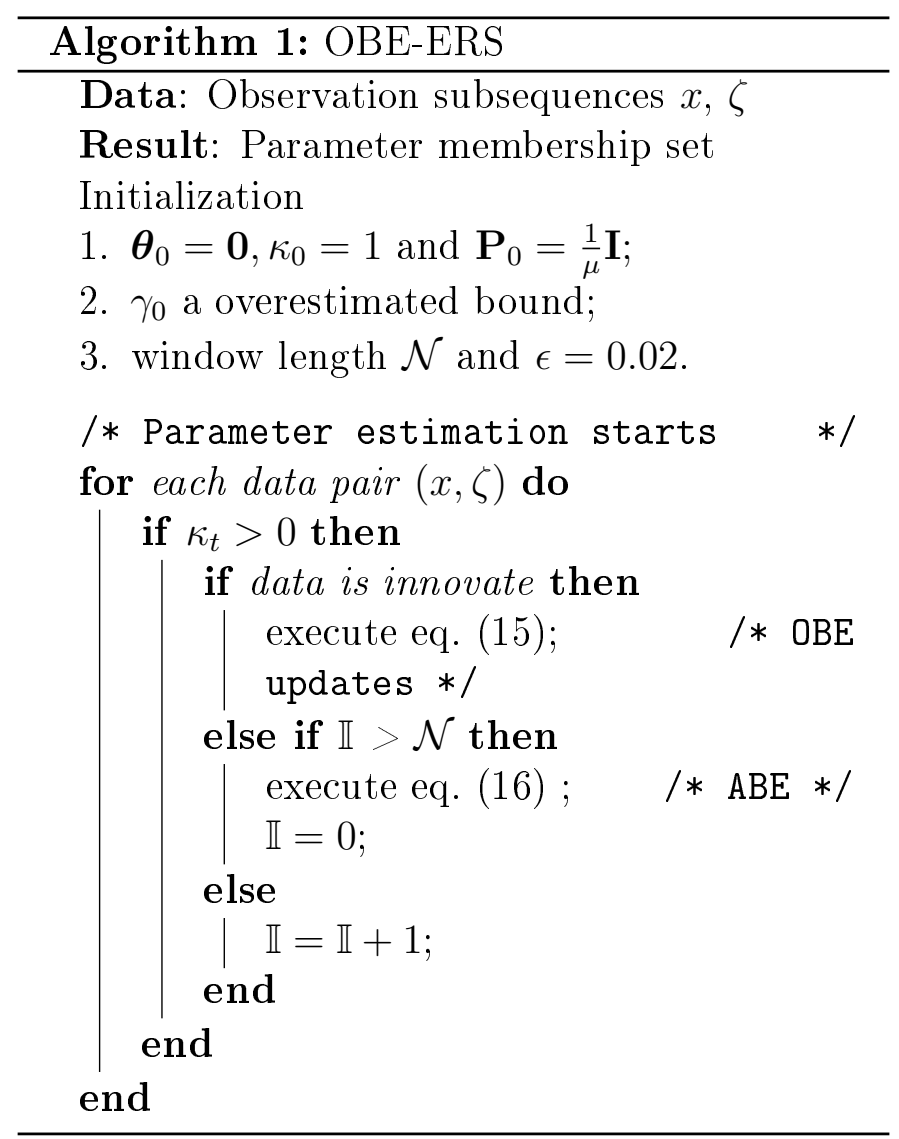

the model. In the next section, these properties characterize the performance of models in the estimation model set, and are exploited by an evolutionary algorithm to guide the exploration of better models.

\section{EVOLUTIONARY MODEL SELECTION}

\section{1. "Cell Biology" of the LTIiP Model}

An evolutionary algorithm is a generic populationbased meta-heuristic optimization algorithm. Falling under the category of evolutionary algorithms, the genetic algorithm solves optimization and search problems using processes inspired by natural evolution [39, 40, 41]. Genetic algorithms often exhibit excellent performance in nonlinear and discrete optimization problems. Here, we use a genetic algorithm to aid in system modeling. The model structure detection problem, which is a NPhard problem, can be mapped coherently into the standard genetic algorithm framework.

In the present formulation, a LTIiP model is an "organism" with a single "chromosome." 
In contrast to the complex chemical structure of chromosomes in living cells, the LTIiP chromosome is a simple, finite, binary sequence in which each bit indicates the presence or absence of a particular "gene." To stretch the analogy, a binary "1" represents a region of "coding $\boldsymbol{D N A}$ " along the model chromosome, while a "0" represents "non-coding $\boldsymbol{D N A}$." Whereas the information in a biological gene is encoded in its sequence of nucleotides, the information in a model gene is merely in its position along the chromosome. In particular, each gene "codes for" a particular regressor function in the model. By analogy, a regressor function, as a "building block" of the model which is indicated by a particular gene, plays the role of a "protein" in the model organism.

Let $\Xi_{\varphi}=\left\{\varphi_{q}\right\}$, of set size $\left|\Xi_{\varphi}\right|$ elements, contain the regressor functions available with which to create models. A chromosome is a string of $\left|\Xi_{\varphi}\right|$ bits which is in one-to-one correspondence with a model. A unity bit in the $\ell^{\text {th }}$ binary position of the chromosome is a "gene" that codes for regressor $\varphi_{q}^{\ell}$ in its model. In principle, then, there are $\left(2^{\left|\Xi_{\varphi}\right|}-1\right)$ different chromosomes, corresponding to an equal number of distinct models. A viable model is one with an effective set of regressor functions and parameter values that allow it to accurately produce the observed $\zeta$ from the observed $x$. Although they appear in the "phenotype," the parameters in some sense represent "regulators of expression" of the genes, the desired expression being the linear mix of proteins that give the model the highest "survival potential." As in biology, survival depends on the inherent suitability of an individual's genetic makeup for meet the challenges of the environment (reflected in $x$ and $\zeta$ ), and also in the realization of that genetic potential resulting from an effective parameter set.

\subsection{Survival Fitness Measures}

The set-theoretic aspects of the identification constrain the sets of parameters to those that are feasible in light of the observations and the error constraints. In turn, they determine the range and statistical viability of phenotypes, and ulti-
Table 1: Adaptation of system modeling to a genetic algorithm

\begin{tabular}{|l|l|}
\hline Cell Biology & System Model \\
\hline Chromosome & LTIiP model \\
\hline Gene & Regressor function \\
\hline Regulator of gene & Parameter \\
\hline
\end{tabular}

mately the plight of the chromosomes. The algorithm starts with a population $\mathbb{P}$, a set of candidate models,

$$
\mathbb{P}[\tau]=\left\{\zeta_{j}^{p}[\tau] \mid \zeta_{j}^{p} \in \mathbb{M}_{\boldsymbol{\theta}, \boldsymbol{\varphi}}\right\}
$$

where $j=1,2, \ldots,|\mathbb{P}|$ indexes individuals in the population, and $\tau=1,2, \ldots$ is the generation index. The parameters of each model is determined by the OBE-ERS algorithm, and the performance of each model (corresponding to a chromosome) is evaluated via a fitness function derived from the set properties of the resulting ellipsoid.

Different fitness functions can be used for model selection [42, 43]. One option is to use the Akaike Final Prediction Error (FPE) [44] as fitness function to evaluate different models. The FPE, which is broadly used for measuring model quality and determining model orders in linear models, is given by

$$
F P E=\frac{N+\sigma Q}{N-\sigma Q} V
$$

where $N$ is the data length, $Q$ is the number of model terms, $\sigma$ is an adjustable parameter, and $V=\frac{1}{N} \sum_{1}^{N}\left(\zeta-\zeta_{j}^{p}\right)^{2}$, which is the sum of squared one-step prediction errors. FPE represents bias and variance trade-offs in model fitting. A useful interpretation of FPE is that the criterion provides a measure by simulating the situation in which the model is tested on a different data set. The goal is to minimize the fitness function defined by FPE criterion.

An alternative quality measure based on the properties of the ellipsoid set is designed empirically:

$$
f=Q \times b^{2} \times R
$$

where $Q$ is the number of regressor signals, $b$ is the estimated bound of the error sequence, and 
$R$ is the ratio of the maximum and the minimum axes of the ellipsoid. The OBE-ERS algorithm starts with an over-estimated error bound, and estimates the error bound recursively. A good model will explain more variations in the data, and approach the 'true' error bound asymptotically as in eq. (8). Conversely, a bad model as in eq. (7) (irrelevant regressors or wrong parameters) will have a larger estimated error bound. In this sense, $b$ serves as a measure of how well a model fits the data. In addition, the parameter hyperellipsoid will approximate a hypersphere if all the chosen regressors contribute equally to the model fitting. Thus, a good model has a smaller $b$ and $R$. $Q$ is added to avoid model overfitting. Notice that both $R$ and $Q$ contribute to the pruning of model parameters. Other fitness functions can also be designed based on the properties of the membership set (e.g., the volume of the ellipsoid).

Each individual is assigned a fitness value derived from the fitness function, which is then used in the selection to bias the new population toward the inclusion of better individuals. Highly-fit individuals (in our case, smaller fitness values) have a high probability of being selected for reproduction. The process continues through subsequent generations. The average fitness of the population improves as more fit individuals appear and interbreed, and the unfit individuals are selected out. The evolutionary algorithm is terminated when a predefined number of generations is reached or the fitness values in the population reaches a prescribed minimum.

\subsection{Evolutionary Operations}

The genetic algorithm begins by creating a random initial population. At each step, the algorithm uses the current population to create the offspring that comprise the next generation. The algorithm selects a group of individuals in the current population, called parents, which contribute their genes to their children. The algorithm usually selects parents that have better fitness values. The operations of selection, crossover, and mutation affect the evolutionary process. Algorithm 2 , evolutionary model selection, outlines this pro- cess, and the details regarding these operators are described as follows. Further details are found in [45, 46].

Selection directs the algorithm towards fitter solutions. Selection must favor fitter candidates over weaker ones. Among different schemes, tournament selection is widely used [40]. The method randomly partitions the populations into "tournaments" and select winners. The winners are the parents which are then used by the crossover and mutation operators to produce children.

Crossover is used to vary the chromosomes from one generation to the next. After selection, parent solutions are chosen to reproduce potentially fitter child solutions with probability $p_{c}$. A unity probability indicates that all the selected chromosomes are used in reproduction. In the uniform crossover [40] scheme, a random binary vector is created by selecting the genes where the vector is a 1 from the first parent, and the genes where the vector is a 0 from the second parent, and combines the genes to form the child.

Mutation is used to introduce random alteration of the genes. It is analogous to biological mutation and typically occurs infrequently. $\mathrm{Mu}-$ tation inverts each bit in a single parent with a low probability $p_{m}$.

Replacement determines which children survive in the next generation. We employ the elite scheme. Elite children are the individuals in the current generation with the best fitness values. These individuals automatically survive to the next generation. The elite children, together with crossover and mutation children, form the next generation.

\section{Experiments and Discussion}

The evolutionary model selection algorithm can be applied to a broad class of identification problems spanning many disciplines. In this section, we report on the use of the method in identifying NARMAX systems. Several factors are common to the experiments. In the initialization of the algorithm, the population size is 50 per generation, the maximum number of iterations $N_{\max }=100$. The crossover rate is $p_{c}=1$ and the mutation rate is $p_{m}=0.1$. For each generation, other than 


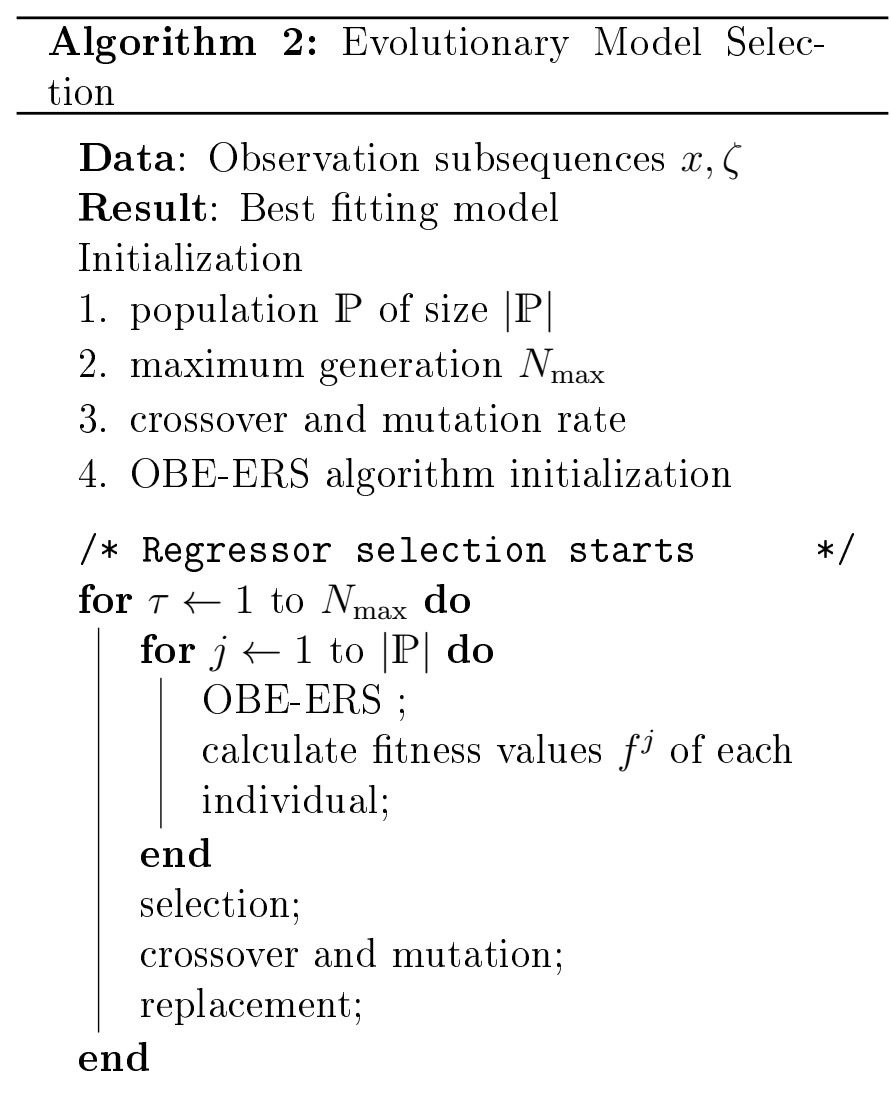

two elite children, $80 \%$ of the children come from crossover, and 20\% from mutation. By design, the candidate set of regressors, $\Xi_{\varphi}$, contains various linear and nonlinear functions. In practice, knowledge of the system can be used to guide the creation of this set, which might include, for example, polynomials and the hyperbolic tangent. For the simulation study in this section, the candidate set contains linear and polynomial nonlinear functions (up to order three, including crossterms) of delayed inputs and outputs with maximum delay of three (i.e. $\zeta[t-1], \zeta[t-2], \zeta[t-$ $3], x[t-1], x[t-2], x[t-3])$, totally 55 , for instance, $x[t-1] \zeta^{2}[t-2]$. Thus, the length of the chromosome is 55 genes, with each bit coding for a regressor, yielding $\left(2^{55}-1\right)$ possible estimation models.

\subsection{System 1}

For simplicity, we begin with a two parameter NARX system

$$
\zeta[t]=x[t-1]+0.25 x[t-2] \zeta[t-3]+e_{*}[t]
$$
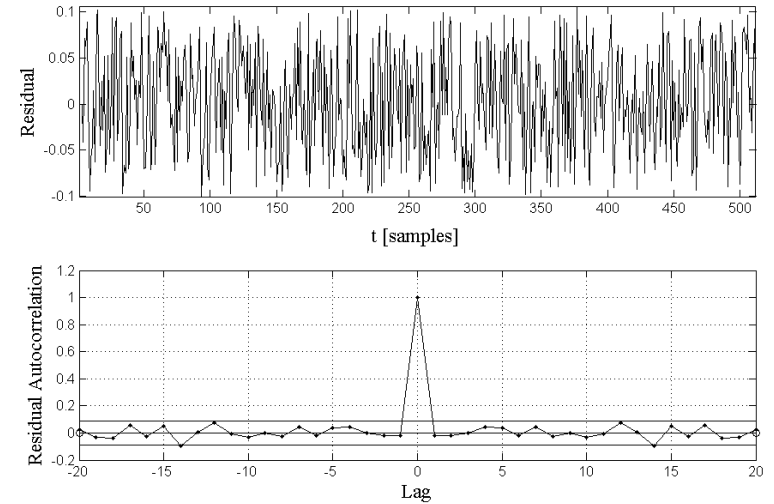

Figure 1: System 1: Residual and linear correlation Test: the horizontal line on the second figure is the $95 \%$ confidence interval. Using the proposed OBE-ERS identification algorithm, the residual appears to be white in accordance with the true system as in eq. (20)

in which the input sequence $x$ is an uncorrelated Gaussian process, $x \sim \mathcal{G}(0,2)$, and $e_{*}[t]$ is uniformlydistributed i.i.d white noise, $e_{*} \sim U(-0.1,0.1)$. The noise is assumed unknown a priori and the noise bound is to be estimated. 1024-point signal observations (input-output pairs) are generated. The observation dataset is divided into a 512point training set and a 512-point test set. The training set is used to identify the model, while the test set is used to assess the performance of the identified model.

Using the FPE criterion of eq. (18) as the fitness function with $\sigma=1$, the estimated model is

$$
\begin{aligned}
\zeta^{p}[t]= & 1.0001 x[t-1]+0.2494 x[t-2] \zeta[t-3] \\
& -0.0009 \zeta[t-1] \zeta[t-2]
\end{aligned}
$$

From the result, we can see that the key structure (the first and second regressor terms) has been detected and the estimated parameters are accurately estimated using the algorithm. The parameter associated with the extra term $\zeta[t-1] \zeta[t-2]$ is so small as to make the term negligible. The residual and the autocorrelation of the residual are shown in Fig. 1, where the residual appears to be white in accordance with the true system as in eq. (20).

The Model Predicted Output (MPO) [18] is 


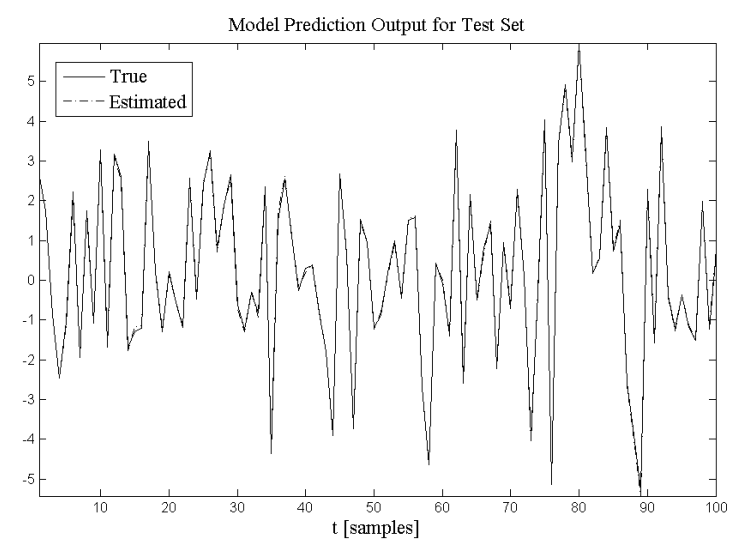

Figure 2: System 1: Identification results: True data (continuous curve) and estimated data (dash-dot curve).For visualization purpose, only 100 samples are shown. The identified model exhibits excellent tracking ability.

calculated using the test set. The system output is initialized by a few measured output values and then MPO is calculated from the identified model driven by only the given input. The result is shown in Fig. 2. For clear visualization, only 100 samples are shown. The selected model exhibits excellent tracking ability. The Pearson correlation coefficient of the true and estimated output is 0.9996 . To further verify that the extra term $-0.0009 \zeta[t-1] \zeta[t-2]$ is negligible, the MPO is calculated with the small term excluded. The correlation coefficient remains 0.9996, which means that the term is insignificant at least to $O\left(10^{-4}\right)$ in this measure.

Using the same setup with only the fitness function changed, another experiment was implemented using the set measure related fitness function of eq. (19). The estimated model is

$$
\begin{aligned}
\zeta^{p}[t]= & 1.0001 x[t-1]+0.2494 x[t-2] \zeta[t-3] \\
& -0.0028 \zeta[t-1] \zeta[t-3]
\end{aligned}
$$

The set property related measure is also successful in identifying the nonlinear system. The correlation coefficient of the original and estimated output is 0.9996 . The result indicates that other than using only the prediction error as conventional point estimate method, the OBE-ERS algorithm provides more useful options as model quality measures and model selection criteria.

\subsection{System 2}

A significant advantage of the OBE estimation is the lack of need for assumptions about stationarity or distributional characteristics of the error sequence. The model selection algorithm can identify the system under different noise conditions, a useful ability for NARMAX model identification with exotic noise conditions.

\subsubsection{Additive Colored Noise}

In this case the system output is contaminated by colored noise, and there are no cross products between the noise and the system input and output measurements. A three parameter system with colored noise is simulated (NARMAX)

$$
\zeta[t]=0.5 \zeta[t-1]+x[t-2]+0.1 x^{2}[t-1]+e_{*}[t]
$$

where colored noise $e_{*}[t]$ is uniformly-distributed i.i.d. white noise sequence $v[t] \sim U(-0.1,0.1)$ by lowpass filtering,

$$
e_{*}[t]=-0.9 e_{*}[t-1]+v[t] .
$$

A 1024-point signal observation (input-output pairs) is generated with 512 points for training and 512 points for testing.

Using the FPE criterion $(\sigma=1)$ as the fitness function, the estimated model is

$$
\begin{aligned}
\zeta^{p}[t]= & 0.5078 \zeta[t-1]+0.9983 x[t-2] \\
& +0.0999 x^{2}[t-1] \\
& +0.0009 \zeta[t-1] \zeta[t-2] x[t-1]
\end{aligned}
$$

The key structure is detected, and very importantly, the estimated parameters are unbiased in the colored noise environment. The residual, as shown in Fig. 3, is colored noise, which corresponds to the true situation. The autocorrelation of the residual measures the linear dependence between the lagged estimated error sequence. The MPO on the 512-point testing dataset is shown in Fig. 4. The correlation coefficient is 0.9938 .

\subsubsection{General Noise}

In the NARMAX model, the noise can be nonlinearly dependent and have some cross terms involving the input and output [18]. A simulated 

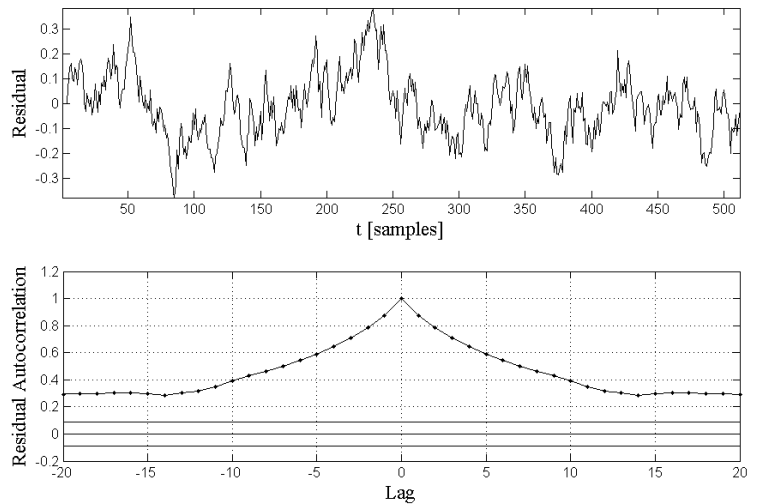

Figure 3: System 2 with colored noise: Residual and linear correlation test: the horizontal line on the second figure is the $95 \%$ confidence interval. The residual appears to be correlated, which is in accordance with the true system as in eq.(24). The algorithm can identify correct models in colored noise scenario.

system to include nonlinear noise terms is as follows:

$$
\begin{aligned}
\zeta[t]= & 0.5 \zeta[t-1]+x[t-2]+0.1 x^{2}[t-1] \\
& +0.5 e_{*}[t-1]+e_{*}[t]+0.1 x[t-1] e_{*}[t-2]
\end{aligned}
$$

Using the FPE criterion $(\sigma=1)$ as the fitness function, the algorithm accurately estimated the model

$\zeta^{p}[t]=0.4970 \zeta[t-1]+0.9980 x[t-2]+0.1007 x^{2}[t-1]$

The residual and autocorrelation are shown in the Fig. 5. The correlation coefficient on the test set is 0.9992 .

The ability to identify the correct model with unbiased parameters without explicit noise modeling makes the algorithm appealing for real system identification, since, in practice, complex noise properties may be unknown.

\subsection{System 3}

A more complex system is simulated to test the algorithm

$$
\begin{aligned}
\zeta[t]= & 0.8 \zeta[t-1]-0.6 \zeta[t-2] \\
& +x[t-1]-0.2 x^{2}[t-1] \\
& +0.01 \zeta[t-1] \zeta[t-2] x[t-2]+e_{*}[t] .
\end{aligned}
$$

As before, 512 points are used for estimation and 512 points for testing. Using the FPE criterion

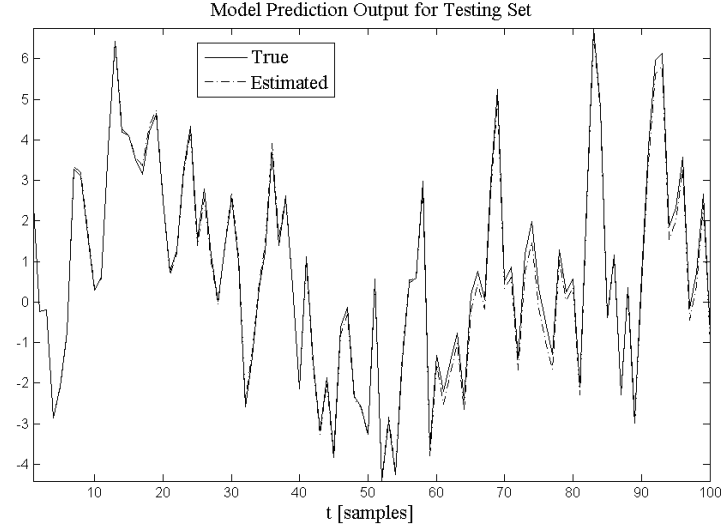

Figure 4: System 2 with colored noise: Identification results: True data (continuous curve) and estimated data (dash-dot curve). The identified model exhibits excellent tracking ability.

( $\sigma=1$ ) as the fitness function, the estimated model is

$$
\begin{aligned}
\zeta^{p}[t]= & 0.8020 \zeta[t-1]-0.6005 \zeta[t-2] \\
& +0.9973 x[t-1]-0.1984 x^{2}[t-1] \\
& +0.0100 \zeta[t-1] \zeta[t-2] x[t-2]
\end{aligned}
$$

Again, the algorithm has correctly identifies the system model. The correlation coefficient on the test set is 0.9997 .

\subsection{System 4}

Finally, to compare the performance of the evolutionary method with some of the most current research, we turn to recent work on Hammerstein systems by Yu, Zhang, and Xie [20]. The improvements in our study as compared to $\mathrm{Yu}$ et. $a l$ are as follows:

- The disturbance in the Hammerstein model used by $\mathrm{Yu}$ et al. is restricted to a stationary white output error, whereas the method of this paper can identify models with much broader classes of noise with no specific distributional or dependence requirements.

- The Hammerstein model is of fixed parametric form with the objective of the $\mathrm{Yu}$ research being the identification of parameter values within this form. The present paper seeks to co-identify the model structure and the parameters within the optimal structure. 

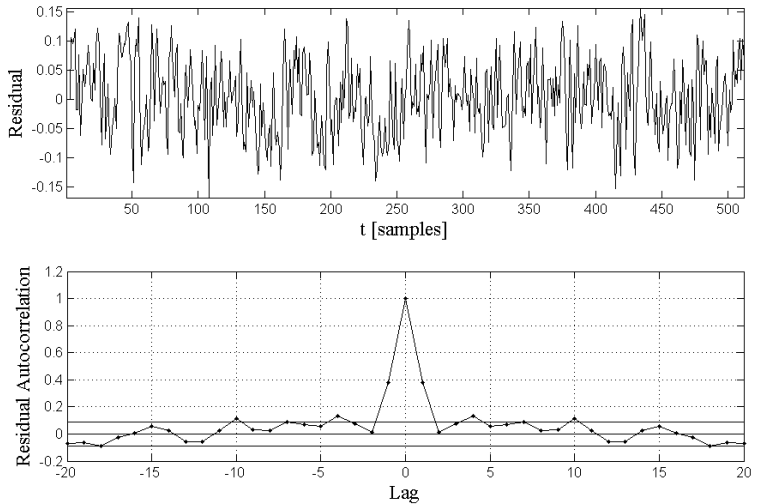

Figure 5: System 2 with general noise: Residual and linear correlation test: the horizontal line on the second figure is the $95 \%$ confidence interval.The residual has the same autocorrelation characteristics as in the true system eq. (26). This example demonstrates the algorithm can identify the correct model even in complex noise conditions.

- In the present work, there is no need for parameter convergence analysis like that appearing in [20, Figs. 4-6] since the parameter convergence is implicit in the set-bounding algorithm. This convergence has been extensively researched in previous papers [31, $38]$.

Each of these factors represent benefits of the present method over the previous work, if the new method can be shown to perform at parity or better on the Hammerstein model relative to the $\mathrm{Yu}$ method. This experiment is designed to test that question.

The model studied by $\mathrm{Yu}$ et al. is a Hammerstein system with a static nonlinearity at the input followed by a linear dynamical system,

$$
\begin{aligned}
z[t]= & 1-0.5 x[t]+0.7 x^{2}[t] \\
\zeta[t]= & 0.3 \zeta[t-1]+0.4 \zeta[t-2] \\
& +z[t]-2.5 z[t-1]+z[t-2]+e_{*}[t]
\end{aligned}
$$

where the system input $x[t]$ and output $\zeta[t]$ are measurable but the intermediate signal $z[t]$ is unmeasurable. The $x[t]$ is an uncorrelated Gaussian process, $x \sim \mathcal{G}(0,2)$, and $e_{*}[t]$ is uniformlydistributed i.i.d white noise. 512-point signal observation is generated for estimation and 512-points for testing. Whereas Yu begins with the known model form, a significant difference in the current work is starting point in which both the model structure and parameters are unknown, both to be determined from the input, output dataset. With some manipulation, eq. (30) can be expressed as the NARX model,

$$
\begin{aligned}
\zeta[t]= & 0.3 \zeta[t-1]+0.4 \zeta[t-2]-0.5 x[t] \\
& +0.7 x^{2}[t]+1.25 x[t-1]-1.75 x^{2}[t-1] \\
& -0.5 x[t-2]+0.7 x^{2}[t-2]-0.5+e_{*}[t]
\end{aligned}
$$

With eq. (31) as the surrogate for Yu's model of eq. (30), the evolutionary model selection algorithm is applied to the dataset. The candidate set is modified to contain linear and polynomial nonlinear functions (up to order three, including cross-terms) of present and delayed inputs, and delayed outputs with maximum delay of two (i.e. $\zeta[t-1], \zeta[t-2], x[t], x[t-1], x[t-2])$. A constant regressor is also included for total of 56 possible terms. Using the FPE criterion $(\sigma=1)$ as the fitness function, the estimated model is

$$
\begin{aligned}
\zeta[t]= & 0.2977 \zeta[t-1]+0.3987 \zeta[t-2]-0.5013 x[t] \\
& +0.6999 x^{2}[t]+1.2490 x[t-1]-1.7492 x^{2}[t-1] \\
& -0.4997 x[t-2]+0.6964 x^{2}[t-2]-0.5056
\end{aligned}
$$

The algorithm has not only correctly estimated the parameter values, but, critically, has properly identified the system model form. The correlation coefficient on the test set is 0.9969 .

Beyond demonstrating the critical advantage of automatic determination of the model form with comparable test set correlation, it should also be noted that the algorithm can be applied when the system is corrupted by correlated or even more complex noise. Asymptotic convergence of the parameter estimation is guaranteed by the OBE algorithm [38] as part of the evolutionary process.

\section{Conclusions}

This paper has suggested a step toward the development of "non-LTI" methods in one important area of SP - parametric model identification. 
A framework has been presented in which nonlinear models can be identified without completely abandoning well-understood and tested methods developed for LTI models.

We first introduced the LTIiP modeling concept in which the signal observations can be (in principle) arbitrarily nonlinear, while the LTIiP property preserves the ability to exploit LTI identification. An LTIiP model can be viewed as a "LTI" model in a world in which the original signals have become morphed into nonlinear combinations that are, nevertheless, if observable, a transformed set of signals. Thus LTI methods can theoretically be used to identify the model parameters in conventional ways. The LTIiP framework, however, has spawned new thinking about model identification, one of the most intenselyresearched areas being that of set-membership based system identification. These methods produce sets of solutions that are consistent with a prior model assumptions, rather than conventional point solutions that result from conventional statistical optimization.

While previous research in conventional and set-theoretic identification has focused on the estimation of parameters, the work presented here simultaneously addresses the selection of the model and the parameter estimation. Measures of quality of set solutions over different configurations of models can be used to assess viability of the underlying models. Models with high "fitness" measures in the set solution space are placed in evolutionary competition with one another in genetic algorithms adapted for the problem setup. The underlying hypothesis of the work is that nonlinear models that are highly tuned to observed signal data will emerge with sufficient opportunity for the models to selectively reproduce and mutate within this competition.

The algorithm has been tested on four varied systems and exhibits excellent performance. As shown in the experiments, the proposed algorithm can identify system models in different noise scenarios. Unlike conventional algorithms, the algorithm can identify NARMAX model without an explicit noise model which is always unknown in the real system. The algorithm has significant potential for further theoretical and applied development.

\section{Reference}

[1] D. T. Westwick, R. E. Kearney, Identification of nonlinear physiological systems, Vol. 7, John Wiley \& Sons, 2003.

[2] H. Khalil, Nonlinear Systems, 3rd Edition, PrenticeHall, 2002.

[3] G. Hinton, L. Deng, D. Yu, G. Dahl, A. Mohamed, N. Jaitly, A. Senior, V. Vanhoucke, P. Nguyen, T. Sainath, et al., Deep neural networks for acoustic modeling in speech recognition: The shared views of four research groups, Signal Processing Magazine, IEEE 29 (6) (2012) 82-97.

[4] A. Carini, G. L. Sicuranza, V. J. Mathews, Efficient adaptive identification of linear-in-the-parameters nonlinear filters using periodic input sequences, Signal Processing 93 (5) (2013) 1210-1220.

[5] A. Kolmogorov, Stationary sequences in Hilbert space, Bulletin of Moscow U. 2 (1941) 1-40.

[6] G. Box, G. Jenkins, Time Series Analysis: Forecasting and Control, 4th Edition, Holden-Day, 1970.

[7] F. Hayashi, Econometrics, Princeton U. Press, 2000.

[8] B. Atal, M. Schroeder, Adaptive predictive coding of speech signals, Bell System Technical J. 49 (1970) 1973-1976.

[9] B. Atal, L. Hanauer, Speech analysis and synthesis by linear prediction of the speech wave, The Journal of the Acoustical Society of America 50 (1971) 637-655.

[10] J. Burg, Maximum entropy spectral analysis, Ph.D. thesis, Stanford U. (1975).

[11] J. Makhoul, Linear prediction: A tutorial review, Proceedings of the IEEE 63 (1975) 561-580.

[12] R. H. Milocco, J. A. De Doná, Robust deconvolution for ARMAX models with gaussian uncertainties, Signal Processing 90 (12) (2010) 3110-3121.

[13] N. Muler, J. Y. Victor, Robust estimation for vector autoregressive models, Computational Statistics \& Data Analysis 65 (2013) 68-79.

[14] A. Mahmoudi, M. Karimi, H. Amindavar, Parameter estimation of autoregressive signals in presence of colored $\operatorname{AR}(1)$ noise as a quadratic eigenvalue problem, Signal Processing 92 (4) (2012) 1151-1156.

[15] C. A. Schmidt, S. I. Biagiola, J. E. Cousseau, J. L. Figueroa, Volterra-type models for nonlinear systems identification, Applied Mathematical Modelling 38 (9) (2014) 2414-2421.

[16] Z. Sigrist, E. Grivel, B. Alcoverro, Estimating secondorder Volterra system parameters from noisy measurements based on an LMS variant or an errorsin-variables method, Signal Processing 92 (4) (2012) 1010-1020.

[17] M. Schetzen, The Volterra and Wiener Theories 
of Nonlinear Systems, Krieger Publishing Company, 2006.

[18] S. A. Billings, Nonlinear System Identification: NARMAX Methods in the Time, Frequency, and Spatiotemporal Domains, John Wiley \& Sons, 2013.

[19] M. Sznaier, Computational complexity analysis of set membership identification of Hammerstein and Wiener systems, Automatica 45 (3) (2009) 701-705.

[20] C. Yu, C. Zhang, L. Xie, A new deterministic identification approach to Hammerstein systems, Signal Processing, IEEE Transactions on 62 (1) (2014) 131140.

[21] I. J. Umoh, T. Ogunfunmi, An affine projection-based algorithm for identification of nonlinear Hammerstein systems, Signal Processing 90 (6) (2010) 2020-2030.

[22] I. J. Leontaritis, S. A. Billings, Input-output parametric models for non-linear systems part i: deterministic non-linear systems, International journal of control 41 (2) (1985) 303-328.

[23] S. Chen, C. F. Cowan, P. M. Grant, Orthogonal least squares learning algorithm for radial basis function networks, Neural Networks, IEEE Transactions on 2 (2) (1991) 302-309.

[24] J. Sjöberg, Q. H. Zhang, L. Ljung, et al., Nonlinear black-box modeling in system identification: A unified overview, Automatica 31 (1995) 1691-1724.

[25] S. S. Chen, D. L. Donoho, M. A. Saunders, Atomic decomposition by basis pursuit, SIAM J. Scientific Computing 20 (1) (1998) 33-61.

[26] E. Walter, J. Norton, H. Piet-Lahanier, M. Milanese (Eds.), Bounding Approaches to System Identification, Perseus Publishing, 1996.

[27] P. L. Combettes, The foundations of set theoretic estimation, Proc. IEEE 81 (1993) 182-208.

[28] J. R. Deller Jr., M. Nayeri, S. F. Odeh, Least-square identification with error bounds for real-time signal processing and control, Proc. IEEE 81 (1993) 815849.

[29] A. Naylor, G. Sell, Linear Operator Theory, HoltRinehart-Winston, 1971.

[30] L. Ljung, System Identification: Theory for the User, 2nd Edition, Prentice-Hall PTR, 1999.

[31] J. R. Deller Jr., M. Nayeri, M. S. Liu, Unifying the landmark developments in optimal bounding ellipsoid identification, International J. Adaptive Control and Signal Processing 8 (1994) 43-60.

[32] E. Walter, H. Piet-Lahanier, Estimation of parameter bounds from bounded-error data: a survey, Mathematics and Computers in Simulation 32 (5) (1990) 449-468.

[33] J. R. Deller Jr., Y. F. Huang, Set-membership identification and filtering for signal processing applications, Circuits, Systems, and Signal Processing 21 (2002) 69-82.

[34] M. Milanese, C. Novara, Unified set membership theory for identification, prediction and filtering of non- linear systems, Automatica 47 (10) (2011) 2141-2151.

[35] R. Arablouei, K. Doğançay, Modified quasi-OBE algorithm with improved numerical properties, Signal Processing 93 (4) (2013) 797-803.

[36] R. Arablouei, K. Doğançay, Steady-state mean squared error and tracking performance analysis of the quasi-OBE algorithm, Signal Processing 93 (1) (2013) 100-108.

[37] J. R. Deller Jr., S. Gollamudi, S. Nagaraj, et al., Convergence analysis of the quasi-OBE algorithm and related performance issues, International J. Adaptive Control and Signal Processing 21 (2007) 499-527.

[38] T. M. Lin, M. Nayeri, J. R. Deller Jr., A consistently convergent OBE algorithm with automatic estimation of error bounds, International J. Adaptive Control and Signal Processing 12 (1998) 305-324.

[39] M. Melanie, An Introduction to Genetic Algorithms, MIT Press, 1998.

[40] C. R. Reeves, J. E. Rowe, Genetic Algorithms: Principles and Perspectives: A Guide to GA Theory, Vol. 20, Springer, 2003.

[41] C. Huang, G. Li, Z. Xu, A. Yu, L. Chang, Design of optimal digital lattice filter structures based on genetic algorithm, Signal Processing 92 (4) (2012) 989998.

[42] S. Khorshidi, M. Karimi, A. R. Nematollahi, New autoregressive (AR) order selection criteria based on the prediction error estimation, Signal Processing 91 (10) (2011) 2359-2370.

[43] M. Karimi, Order selection criteria for vector autoregressive models, Signal Processing 91 (4) (2011) 955969.

[44] H. Akaike, Fitting autoregressive models for prediction, Annals of the Institute of Statistical Mathematics 21 (1) (1969) 243-247.

[45] J. Yan, J. R. Deller Jr., D. B. Fleet, et al., Evolutionary identification of nonlinear parametric models with a set-theoretic fitness criterion, in: Proc. 2013 IEEE China Summit and International Conf. Signal and Information Processing, Vol. 1, 2013, pp. 44-48.

[46] J. Yan, J. R. Deller Jr., M. Yao, E. D. Goodman, Evolutionary model selection for identification of nonlinear parametric systems, in: Proc. 2014 IEEE China Summit and International Conf. Signal and Information Processing, 2014, pp. 693-697. 